\title{
Scheduling Jobs with Variable Job Processing Times on Unrelated Parallel Machines
}

\author{
Guang-Qian Zhang, Jian-Jun Wang, and Ya-Jing Liu \\ Faculty of Management and Economics, Dalian University of Technology, Dalian 116024, China \\ Correspondence should be addressed to Jian-Jun Wang; wangjianjun_2008@163.com
}

Received 21 March 2014; Accepted 30 April 2014; Published 26 May 2014

Academic Editor: Chin-Chia Wu

Copyright (C) 2014 Guang-Qian Zhang et al. This is an open access article distributed under the Creative Commons Attribution License, which permits unrestricted use, distribution, and reproduction in any medium, provided the original work is properly cited.

$m$ unrelated parallel machines scheduling problems with variable job processing times are considered, where the processing time of a job is a function of its position in a sequence, its starting time, and its resource allocation. The objective is to determine the optimal resource allocation and the optimal schedule to minimize a total cost function that dependents on the total completion (waiting) time, the total machine load, the total absolute differences in completion (waiting) times on all machines, and total resource cost. If the number of machines is a given constant number, we propose a polynomial time algorithm to solve the problem.

\section{Introduction}

In classical scheduling theory, it is assumed that the job processing times are fixed and constant values. In practice, however, we often encounter settings in which job processing times may be subject to change due to the deterioration effects and/or learning effects and/or controllable processing times. Extensive surveys of different scheduling models and problems involving deteriorating jobs can be found in Alidaee and Womer [1], Cheng et al. [2], and Gawiejnowicz [3]. A recent survey of the scheduling problems with learning effects could be found in Biskup [4]. A recent survey of the scheduling problems with controllable processing times was given by Shabtay and Steiner [5]. More recent papers that have considered scheduling problems with deterioration effects and/or learning effects and/or controllable processing times include Bai et al. [6, 7], Cheng et al. [8], Gorczyca and Janiak [9], Hsu and Yang [10], Huang and Wang [11], Lee et al. [12], Leyvand et al. [13], Nian and Mao [14], Shabtay and Steiner [15], Wang et al. [16], J. B. Wang and M. Z. Wang [17], J. B. Wang and J. J. Wang [18], Wang et al. [19-21], Wei et al. [22], Wu and Lee [23], Wu and Liu [24], Yang and Kuo [25], S. J. Yang and D. L. Yang [26], Yang et al. [27], Yin et al.
[28-31], and Zhao and Tang [32]. Mosheiov and Sidney [33] considered the single machine model:

$$
p_{j}=a_{j} r^{b_{j}},
$$

where $a_{j}$ is the original (normal) processing time of job $J_{j}$, $p_{j}$ is the actual processing time of job $J_{j}, r$ is the position of job $J_{j}$ when scheduled on the machine, and $b_{j} \leq 0$ is the job-dependent learning index of job $J_{j}$. Yang and Kuo [25] considered single machine scheduling with the deterioration and learning effect in which the job processing times are

$$
p_{j}=a_{j} r^{b}+\alpha t
$$

where $t$ is the starting time of job $J_{j}, b \leq 0$ is a learning index, and $\alpha \geq 0$ is a common deterioration rate for all the jobs. Shabtay and Steiner [15] considered single machine scheduling with the resource allocation model in which the job processing times are

$$
p_{j}=a_{j}-\beta_{j} u_{j},
$$

where $u_{j}$ is the amount of a nonrenewable resource allocated to job $J_{j}$, with $0 \leq u_{j} \leq \bar{u}_{j}<a_{j} / \beta_{j}$, where $\bar{u}_{j}$ denote 
the maximum amount of resource allocated to job $J_{j}$ and $\beta_{j}$ is the positive compression rate of job $J_{j}$.

Wang et al. [19] considered single machine scheduling problem where the job processing time is a function of position in a sequence, starting time, and resource allocation to this job on a single machine; that is, the model

$$
p_{j}=a_{j} r^{b}+\alpha t-\beta_{j} u_{j}
$$

where $0 \leq u_{j} \leq \bar{u}_{j}<a_{j} n^{b} / \beta_{j}$. For minimizing a cost function containing makespan, total completion (waiting) time, total absolute differences in completion (waiting) times, and total resource cost, they proved that the problem can be solved in polynomial time.

Wang et al. [19] considered single machine scheduling problem with effect of deterioration, learning, and resource allocation simultaneously. The example of the phenomena of deterioration, learning effect, and resource allocation occurring simultaneously can be found in Wang et al. [19]. However, the parallel machine scheduling is interesting and closer to real problems in practice (Cheng et al. [8], Hsu and Yang [10], Huang and Wang [11], Yang et al. [27], and Yin et al. [30]). This paper extends the single machine scheduling results of Wang et al. [19], by considering unrelated parallel machine scheduling problems that include the one given in Wang et al. [19] as a special case. The remainder of this paper is organized as follows. In Section 2 we formulate the model. In Sections 3 and 4, we show that the problem can be solved in polynomial time for two different cost functions. The last section contains some conclusions.

\section{Problem Formulation}

The model is described as follows. There are $n$ independent jobs $\left\{J_{1}, J_{2}, \ldots, J_{n}\right\}$ to be processed on $m$ unrelated parallel machines $\left\{M_{1}, M_{2}, \ldots, M_{m}\right\}$. Each of them is available at time 0 . The machine can handle one job at a time, and preemption is not allowed. Let $n_{i}$ denote the number of jobs assigned to $M_{i}(i=1,2, \ldots, m)$ and $P(n, m)=\left(n_{1}, n_{2}, \ldots, n_{m}\right)$ denote a job-allocation vector, where $\left(n_{1}+n_{2}+\cdots n_{m}=n\right)$. We assume, as in most practical situations, that $m<n$ and $m$ are a given constant. Each job can be processed on any one of the $m$ unrelated parallel machines. Associated with each job $J_{j}(j=1,2, \ldots, n)$ on machine $M_{i}$, there is a normal processing time $a_{i j}$. Let $p_{i j}$ denote the actual processing time for job $J_{j}$ on machine $M_{i}$. In this paper, we consider a general unrelated parallel machines model stemming from Yang and Kuo [25], Shabtay and Steiner [15], and Wang et al. [19]; that is,

$$
p_{i j}=a_{i j} r^{b_{i j}}+\alpha t-\theta_{i j} u_{i j}
$$

where $r$ is the position of job $J_{j}$ when scheduled on machine $M_{i}, t$ is the starting time of job $J_{j}$ on machine $M_{i}, b_{i j} \leq 0$ is a job-dependent learning index, $\alpha \geq 0$ is a common deterioration rate for all the jobs, $\theta_{i j} \geq 0$ is the positive compression rate of job $J_{j}$ on machine $M_{i}$, and $u_{i j}$ is the amount of resource that can be allocated to job $J_{j}$ on machine $M_{i}$, with $0 \leq u_{i j} \leq \bar{u}_{i j}<a_{i j} n^{b_{i j}} / \theta_{i j}$, where $\bar{u}_{i j}$ is the upper bound on the amount of resource that can be allocated to job $J_{j}$ on machine $M_{i}$.

Let $J_{i[j]}$ denote the $j$ th job on machine $M_{i}$ and $C_{i[j]}\left(W_{i[j]}\right)$ denote the completion (waiting) time of job $J_{i[j]} ; p_{i[j]}, a_{i[j]}$, $b_{i[j]}, \theta_{i[j]}, u_{i[j]}$, and $\bar{u}_{i[j]}$ are defined similarly, where $W_{i[j]}=$ $C_{i[j]}-p_{i[j]}$. Let $L_{i}=\max \left\{C_{i j} \mid j=1,2, \ldots, n_{i}\right\}, T C_{i}=$ $\sum_{j=1}^{n_{i}} C_{i j}\left(T W_{i}=\sum_{j=1}^{n_{i}} W_{i j}\right)$, and $T A D C_{i}=\sum_{j=1}^{n_{i}} \sum_{l=j}^{n_{i}} \mid C_{i j}-$ $C_{i l} \mid\left(T A D W_{i}=\sum_{j=1}^{n_{i}} \sum_{l=j}^{n_{i}}\left|W_{i j}-W_{i l}\right|\right)$ be the load, the total completion (waiting) times, and the total absolute differences in completion (waiting) times of machine $M_{i}$. Then, the total machine load, the total completion (waiting) time, and the total absolute deviation of job completion (waiting) time on all machines are $\sum_{i=1}^{m} L_{i}, \sum_{i=1}^{m} T C_{i}\left(\sum_{i=1}^{m} T W_{i}\right)$, and $\sum_{i=1}^{m} T_{A D C_{i}}\left(\sum_{i=1}^{m} T A D W_{i}\right)$, respectively. The objective is to determine the optimal resource allocations and the optimal schedule on the machines so that the corresponding value of the following cost functions is optimal:

$$
\begin{aligned}
Z_{1}(\pi, u)= & \alpha_{1} \sum_{i=1}^{m} L_{i}+\alpha_{2} \sum_{i=1}^{m} T C_{i}+\alpha_{3} \sum_{i=1}^{m} T A D C_{i} \\
& +\alpha_{4} \sum_{i=1}^{m} \sum_{j=1}^{n_{i}} G_{i j} u_{i j}, \\
Z_{2}(\pi, u)= & \alpha_{1} \sum_{i=1}^{m} L_{i}+\alpha_{2} \sum_{i=1}^{m} T W_{i}+\alpha_{3} \sum_{i=1}^{m} T A D W_{i} \\
& +\alpha_{4} \sum_{i=1}^{m} \sum_{j=1}^{n_{i}} G_{i j} u_{i j},
\end{aligned}
$$

where weights $\alpha_{1} \geq 0, \alpha_{2} \geq 0, \alpha_{3} \geq 0$, and $\alpha_{4} \geq 0$ are given constants and $G_{i j}$ is the per time unit cost associated with the resource allocation. Using the three-field notation introduced by Graham et al. [34], the corresponding scheduling problem is denoted by $R m\left|p_{i j}=a_{i j} r^{b_{i j}}+\alpha t-\theta_{i j} u_{i j}\right| \alpha_{1} \sum_{i=1}^{m} L_{i}+$ $\alpha_{2} \sum_{i=1}^{m} T C_{i}+\alpha_{3} \sum_{i=1}^{m} T_{A D C_{i}}+\alpha_{4} \sum_{i=1}^{m} \sum_{j=1}^{n_{i}} G_{i j} u_{i j}$ and $R m\left|p_{i j}=a_{i j} r^{b_{i j}}+\alpha t-\theta_{i j} u_{i j}\right| \alpha_{1} \sum_{i=1}^{m} L_{i}+\alpha_{2} \sum_{i=1}^{m} T W_{i}+$ $\alpha_{3} \sum_{i=1}^{m} T A D W_{i}+\alpha_{4} \sum_{i=1}^{m} \sum_{j=1}^{n_{i}} G_{i j} u_{i j}$.

$$
\begin{aligned}
& \text { 3. Problem } R m\left|p_{i j}=a_{i j} r^{b_{i j}}+\alpha t-\theta_{i j} u_{i j}\right| \\
& \alpha_{1} \sum_{i=1}^{m} L_{i}+\alpha_{2} \sum_{i=1}^{m} T C_{i}+\alpha_{3} \sum_{i=1}^{m} T A D C_{i}+ \\
& \alpha_{4} \sum_{i=1}^{m} \sum_{j=1}^{n_{i}} G_{i j} u_{i j}
\end{aligned}
$$

If the number of jobs on machine $M_{i}$ is known in advance, from Wang et al. [19], we have

$$
\begin{aligned}
C_{i[j]}= & \sum_{l=1}^{j}(1+\alpha)^{j-l}\left(a_{i[l]} l^{b_{i[l]}}-\beta_{i[l]} u_{i[l]}\right), \\
p_{i[j]}= & a_{i[j]} j^{b_{i[j]}}-\beta_{i[j]} u_{i[j]} \\
& +\alpha \sum_{l=1}^{j-1}(1+\alpha)^{j-1-l}\left(a_{i[l]} l^{b_{i[l]}}-\beta_{i[l]} u_{i[l]}\right) .
\end{aligned}
$$


From Kanet [35], we have $\operatorname{TADC}_{i}=\sum_{j=1}^{n_{i}}(j-1)\left(n_{i}-\right.$ $j+1) p_{i[j]}$; in addition, $C_{i[j]}=\sum_{l=1}^{j} p_{i[l]}, L_{i}=\sum_{j=1}^{n_{i}} p_{i[j]}$, and $T C_{i}=\sum_{j=1}^{n_{i}} C_{i[j]}=\sum_{j=1}^{n_{i}}\left(n_{i}-j+1\right) p_{i[j]}$; hence, we have

$$
\begin{aligned}
Z_{1}(\pi, u) & \alpha_{1} \sum_{i=1}^{m} \sum_{j=1}^{n_{i}} p_{i[j]}+\alpha_{2} \sum_{i=1}^{m} \sum_{j=1}^{n_{i}}\left(n_{i}-j+1\right) p_{i[j]} \\
& +\alpha_{3} \sum_{i=1}^{m} \sum_{j=1}^{n_{i}}(j-1)\left(n_{i}-j+1\right) p_{i[j]} \\
& +\alpha_{4} \sum_{i=1}^{m} \sum_{j=1}^{n_{i}} G_{i[j]} u_{i[j]} \\
= & \sum_{i=1}^{m} \sum_{j=1}^{n_{i}}\left[\alpha_{1}+\alpha_{2}\left(n_{i}+1-j\right)+\alpha_{3}(j-1)\left(n_{i}-j+1\right)\right] p_{i[j]} \\
& +\alpha_{4} \sum_{i=1}^{m} \sum_{j=1}^{n_{i}} G_{i[j]} u_{i[j]} \\
= & \sum_{i=1}^{m} \sum_{j=1}^{n_{i}} \omega_{i j} p_{i[j]}+\alpha_{4} \sum_{i=1}^{m} \sum_{j=1}^{n_{i}} G_{i[j]} u_{i[j]} \\
= & \sum_{i=1}^{m} \sum_{j=1}^{n_{i}} \omega_{i j}\left(a_{i[j]} j^{b_{i[j]}}-\beta_{i[j]} u_{i[j]}\right. \\
& \left.+\alpha \sum_{l=1}^{j-1}(1+\alpha)^{j-1-l}\left(a_{i[l]} l_{i[l]}^{b^{\prime}}-\beta_{i[l]} u_{i[l]}\right)\right)
\end{aligned}
$$$$
+\alpha_{4} \sum_{i=1}^{m} \sum_{j=1}^{n_{i}} G_{i[j]} u_{i[j]}
$$$$
=\sum_{i=1}^{m}\left[\omega_{i 1}\left(a_{i[1]}\right]^{b_{i[1]}}-\beta_{i[1]} u_{i[1]}\right)
$$$$
+\omega_{i 2}\left(a_{i[2]} 2^{b_{i[2]}}-\beta_{i[2]} u_{i[2]}\right.
$$$$
\left.+\alpha\left(a_{i[1]} 1^{b_{i[1]}}-\beta_{i[1]} u_{i[1]}\right)\right)
$$$$
+\omega_{i 3}\left(a_{i[3]} 3^{b_{i[3]}}-\beta_{i[3]} u_{i[3]}\right.
$$$$
+\alpha\left(a_{i[2]} 2^{b_{i[2]}}-\beta_{i[2]} u_{i[2]}+(1+\alpha)\right.
$$$$
\left.\left.\left.\times\left(a_{i[1]}\right]^{b_{i[1]}}-\beta_{i[1]} u_{i[1]}\right)\right)\right)
$$$$
+\omega_{i 4}\left(a_{i[4]} 4^{b_{i[4]}}-\beta_{i[4]} u_{i[4]}\right.
$$$$
+\alpha\left(a_{i[3]} 3^{b_{i[3]}}-\beta_{i[3]} u_{i[3]}+(1+\alpha)\right.
$$$$
\times\left(a_{i[2]} 2^{b_{i[2]}}-\beta_{i[2]} u_{i[2]}\right)
$$$$
\left.\left.+(1+\alpha)^{2}\left(a_{i[1]} 1^{b_{i[1]}}-\beta_{i[1]} u_{i[1]}\right)\right)\right)+\cdots
$$$$
+\omega_{i, n_{i}-1}\left(a_{i\left[n_{i}-1\right]}\left(n_{i}-1\right)^{b_{i\left[n_{i}-1\right]}}-\beta_{i\left[n_{i}-1\right]} u_{i\left[n_{i}-1\right]}\right.
$$$$
+\alpha\left(a_{i\left[n_{i}-2\right]}\left(n_{i}-2\right)^{b_{i\left[n_{i}-2\right]}}\right.
$$$$
-\beta_{i\left[n_{i}-2\right]} u_{i\left[n_{i}-2\right]}+(1+\alpha)
$$$$
\times\left(a_{i\left[n_{i}-3\right]}\left(n_{i}-3\right)^{b_{i\left[n_{i}-3\right]}}\right.
$$$$
\left.-\beta_{i\left[n_{i}-3\right]} u_{i\left[n_{i}-3\right]}\right)+\cdots
$$$$
+(1+\alpha)^{n_{i}-4}\left(a_{i[2]} 2^{b_{i[2]}}-\beta_{i[2]} u_{i[2]}\right)
$$$$
\left.\left.+(1+\alpha)^{n_{i}-3}\left(a_{i[1]} 1^{b_{i[1]}}-\beta_{i[1]} u_{i[1]}\right)\right)\right)
$$$$
+\omega_{i n_{i}}\left(a_{i\left[n_{i}\right]} n_{i}^{b_{i\left[n_{i}\right]}}-\beta_{i\left[n_{i}\right]} u_{i\left[n_{i}\right]}\right.
$$$$
+\alpha\left(a_{i\left[n_{i}-1\right]}\left(n_{i}-1\right)^{b_{i\left[n_{i}-1\right]}}-\beta_{i\left[n_{i}-1\right]} u_{i\left[n_{i}-1\right]}\right.
$$$$
+(1+\alpha)\left(a_{i\left[n_{i}-2\right]}\left(n_{i}-2\right)^{b_{i\left[n_{i}-2\right]}}\right.
$$$$
\left.-\beta_{i\left[n_{i}-2\right]} u_{i\left[n_{i}-2\right]}\right)+\cdots
$$$$
+(1+\alpha)^{n_{i}-3}\left(a_{i[2]} 2^{b_{i[2]}}-\beta_{i[2]} u_{i[2]}\right)
$$$$
\left.\left.\left.+(1+\alpha)^{n_{i}-2}\left(a_{i[1]} 1^{b_{i[1]}}-\beta_{i[1]} u_{i[1]}\right)\right)\right)\right]
$$

$$
\begin{aligned}
& +\alpha_{4} \sum_{i=1}^{m} \sum_{j=1}^{n_{i}} G_{i[j]} u_{i[j]} \\
& =\sum_{i=1}^{m}\left[\left(\omega_{i 1}+\alpha \omega_{i 2}+\alpha(1+\alpha) \omega_{i 3}+\cdots+\alpha(1+\alpha)^{n-2} \omega_{i n_{i}}\right)\right. \\
& \quad \times\left(a_{i[1]} 1^{b_{i[1]}}-\beta_{i[1]} u_{i[1]}\right) \\
& +\left(\omega_{i 2}+\alpha \omega_{i 3}+\alpha(1+\alpha) \omega_{i 4}+\cdots\right. \\
& \left.\quad+\alpha(1+\alpha)^{n_{i}-3} \omega_{i n_{i}}\right)\left(a_{i[2]} 2^{b_{i[2]}}-\beta_{i[2]} u_{i[2]}\right) \\
& +\left(\omega_{i 3}+\alpha \omega_{i 4}+\alpha(1+\alpha) \omega_{i 5}+\cdots\right. \\
& \left.\quad+\alpha(1+\alpha)^{n_{i}-4} \omega_{i n_{i}}\right)\left(a_{i[3]} 3^{b_{i[3]}}-\beta_{i[3]} u_{i[3]}\right) \\
& +\cdots+\left(\omega_{i, n_{i}-1}+\alpha \omega_{i n_{i}}\right)\left(a_{i\left[n_{i}-1\right]}-\beta_{i\left[n_{i}-1\right]} u_{i\left[n_{i}-1\right]}\right) \\
& \left.+\omega_{i n_{i}}\left(a_{i\left[n_{i}\right]} n_{i}^{b_{i\left[n_{i}\right]}}-\beta_{i\left[n_{i}\right.} u_{i\left[n_{i}\right]}\right)\right] \\
& +\alpha_{4} \sum_{i=1}^{m} \sum_{j=1}^{n_{i}} G_{i[j]} u_{i[j]} \\
& =\sum_{i=1}^{m} \sum_{j=1}^{n_{i}} \Omega_{i j} a_{i[j]} b^{b_{i[j]}}+\sum_{i=1}^{m} \sum_{j=1}^{n_{i}}\left(\alpha_{4} G_{i[j]}-\beta_{i[j]} \Omega_{i j}\right) u_{i[j]},
\end{aligned}
$$


where $\omega_{i j}=\alpha_{1}+\alpha_{2}\left(n_{i}+1-j\right)+\alpha_{3}(j-1)\left(n_{i}-j+1\right)$ and

$$
\begin{aligned}
\Omega_{i 1} & =\omega_{i 1}+\alpha \omega_{i 2}+\alpha(1+\alpha) \omega_{i 3}+\cdots+\alpha(1+\alpha)^{n_{i}-2} \omega_{i n_{i}}, \\
\Omega_{i 2} & =\omega_{i 2}+\alpha \omega_{i 3}+\alpha(1+\alpha) \omega_{i 4}+\cdots+\alpha(1+\alpha)^{n_{i}-3} \omega_{i n_{i}}, \\
\Omega_{i 3} & =\omega_{i 3}+\alpha \omega_{i 4}+\alpha(1+\alpha) \omega_{i 5}+\cdots+\alpha(1+\alpha)^{n_{i}-4} \omega_{i n_{i}}, \\
& \vdots \\
\Omega_{i, n_{i}-1} & =\omega_{i, n_{i}-1}+\alpha \omega_{i n_{i}}, \\
\Omega_{i n_{i}} & =\omega_{i n_{i}} .
\end{aligned}
$$

From (9), for any given sequence on all machines, the optimal resource allocation on all machines can be obtained by the following.

Lemma 1. For a given sequence, the optimal resource allocation of the problem $R m\left|p_{i j}=a_{i j} r^{b_{i j}}+\alpha t-\theta_{i j} u_{i j}\right| \alpha_{1} \sum_{i=1}^{m} L_{i}+$ $\alpha_{2} \sum_{i=1}^{m} T C_{i}+\alpha_{3} \sum_{i=1}^{m} T A D C_{i}+\alpha_{4} \sum_{i=1}^{m} \sum_{j=1}^{n_{i}} G_{i j} u_{i j}$ can be determined by

$$
u_{i[j]}^{*}= \begin{cases}\bar{u}_{i[j]}, & \text { if } \alpha_{4} G_{i[j]}-\beta_{i[j]} \Omega_{i j}<0, \\ u_{i[j]} \in\left[0, \bar{u}_{i[j]}\right], & \text { if } \alpha_{4} G_{i[j]}-\beta_{i[j]} \Omega_{i j}=0, \\ 0, & \text { if } \alpha_{4} G_{i[j]}-\beta_{i[j]} \Omega_{i j}>0,\end{cases}
$$

where $u_{i[j]}^{*}, i=1,2, \ldots, m ; j=1,2, \ldots, n_{i}$, represents the optimal resource allocation of the job in position $j$ on machine $M_{i}$.

Proof. For the problem $R m\left|p_{i j}=a_{i j} r^{b_{i j}}+\alpha t-\theta_{i j} u_{i j}\right|$ $\alpha_{1} \sum_{i=1}^{m} L_{i}+\alpha_{2} \sum_{i=1}^{m} T C_{i}+\alpha_{3} \sum_{i=1}^{m} T A D C_{i}+\alpha_{4} \sum_{i=1}^{m} \sum_{j=1}^{n_{i}} G_{i j} u_{i j}$, taking the derivative by $u_{i[j]}$ to (9), we have $d f(\pi, u) / u_{i[j]}=\alpha_{4} G_{i[j]}-\beta_{i[j]} \Omega_{i j}$ for $i=1,2, \ldots, m$; $j=1,2, \ldots, n_{i}$. Hence, if $\alpha_{4} G_{i[j]}-\beta_{i[j]} \Omega_{i j}>0$, we should not allocate any resource to job $J_{i[j]}$; if $\alpha_{4} G_{i[j]}-\beta_{i[j]} \Omega_{i j}<0$, we will allocate the maximal feasible amount of resource to job $J_{i[j]}$; and if $\alpha_{4} G_{i[j]}-\beta_{i[j]} \Omega_{i j}=0$, any feasible resource allocation can be optimal.

We define $x_{i j r}=1$ if job $J_{j}$ is scheduled in position $r$ on machine $M_{i}$, and $x_{i j r}=0$ otherwise. If the number of jobs on machine $M_{i}$ is known in advance, then we formulate the $R m\left|p_{i j}=a_{i j} r^{b_{i j}}+\alpha t-\theta_{i j} u_{i j}\right| \alpha_{1} \sum_{i=1}^{m} L_{i}+\alpha_{2} \sum_{i=1}^{m} T C_{i}+$ $\alpha_{3} \sum_{i=1}^{m} T A D C_{i}+\alpha_{4} \sum_{i=1}^{m} \sum_{j=1}^{n_{i}} G_{i j} u_{i j}$ problem as the following assignment problem:

$$
\operatorname{Min} Z=\sum_{i=1}^{m} \sum_{r=1}^{n_{i}} \sum_{j=1}^{n} \lambda_{i j r} x_{i j r}
$$

subject to

$$
\begin{gathered}
\sum_{i=1}^{m} \sum_{r=1}^{n_{i}} x_{i j r}=1, \quad j=1,2, \ldots, n, \\
\sum_{j=1}^{n} x_{i j r}=1, \quad i=1,2, \ldots, m ; r=1,2, \ldots, n_{i}, \\
x_{i j r}=0 \text { or } 1, \quad i=1,2, \ldots, m ; \\
r=1,2, \ldots, n_{i} ; \quad j=1,2, \ldots, n,
\end{gathered}
$$

where

$$
\begin{aligned}
& \lambda_{i j r} \\
& = \begin{cases}\Omega_{i r} a_{i j} r^{b_{i j}}, & \text { if } \alpha_{4} G_{i j}-\beta_{i j} \Omega_{i r} \geq 0, \\
\Omega_{i r} a_{i j} r^{b_{i j}}+\left(\alpha_{4} G_{i j}-\beta_{i j} \Omega_{i r}\right) \bar{u}_{i j}, & \text { if } \alpha_{4} G_{i j}-\beta_{i j} \Omega_{i r}<0 .\end{cases}
\end{aligned}
$$

Constraint (13) makes sure that each job is scheduled exactly once. Constraint (14) makes sure that each position on each machine is taken by one job.

Next, the question is how many $P(n, m)=\left(n_{1}, n_{2}, \ldots, n_{m}\right)$ vectors exist. Note that $n_{i}$ may be $0,1,2, \ldots, n$ for $i=1$, $2, \ldots, m$. So, if we get the numbers of jobs on the first $m-1$ machines, the number of jobs processed on the last machine is then determined uniquely due to $n_{1}+n_{2}+\cdots+n_{m}=n$. Therefore, the upper bound of the number of $P(n, m)$ vectors is $(n+1)^{m-1}$. Based on the above analysis, we have the following result.

Theorem 2. The problem $R m\left|p_{i j}=a_{i j} r^{b_{i j}}+\alpha t-\theta_{i j} u_{i j}\right|$ $\alpha_{1} \sum_{i=1}^{m} L_{i}+\alpha_{2} \sum_{i=1}^{m} T C_{i}+\alpha_{3} \sum_{i=1}^{m} T_{A D C}+\alpha_{4} \sum_{i=1}^{m} \sum_{j=1}^{n_{i}} G_{i j} u_{i j}$ can be solved in $\mathrm{O}\left(n^{m+2}\right)$ time; that is, the problem is polynomially solvable because $m$ is a constant.

Base on the above analysis, we can determine the optimal solution for the problem $R m \mid p_{i j}=a_{i j} r^{b_{i j}}+\alpha t-$ $\theta_{i j} u_{i j} \mid \alpha_{1} \sum_{i=1}^{m} L_{i}+\alpha_{2} \sum_{i=1}^{m} T C_{i}+\alpha_{3} \sum_{i=1}^{m} T A D C_{i}+$ $\alpha_{4} \sum_{i=1}^{m} \sum_{j=1}^{n_{i}} G_{i j} u_{i j}$ via the following algorithm.

Algorithm 3. Consider the following.

Step 1. For all the possible vectors $\left(n_{1}, n_{2}, \ldots, n_{m}\right)$, solve the assignment problems ((12)-(16)). Then, obtain the optimal schedule and the corresponding total cost $\alpha_{1} \sum_{i=1}^{m} L_{i}+$ $\alpha_{2} \sum_{i=1}^{m} T C_{i}+\alpha_{3} \sum_{i=1}^{m} T A D C_{i}+\alpha_{4} \sum_{i=1}^{m} \sum_{j=1}^{n_{i}} G_{i j} u_{i j}$ for each possible vector $\left(n_{1}, n_{2}, \ldots, n_{m}\right)$.

Step 2. The optimal solution for the problem is the one with the minimum value of the total cost $\alpha_{1} \sum_{i=1}^{m} L_{i}+\alpha_{2} \sum_{i=1}^{m} T C_{i}+$ $\alpha_{3} \sum_{i=1}^{m} T A D C_{i}+\alpha_{4} \sum_{i=1}^{m} \sum_{j=1}^{n_{i}} G_{i j} u_{i j}$.

Step 3. Calculate the optimal resources allocation by using (11). 
The following example illustrates the working of Algorithm 3 for the problem $R m\left|p_{i j}=a_{i j} r^{b_{i j}}+\alpha t-\theta_{i j} u_{i j}\right|$ $\alpha_{1} \sum_{i=1}^{m} L_{i}+\alpha_{2} \sum_{i=1}^{m} T C_{i}+\alpha_{3} \sum_{i=1}^{m} T A D C_{i}+\alpha_{4} \sum_{i=1}^{m} \sum_{j=1}^{n_{i}} G_{i j} u_{i j}$.

Example 4. Data. $n=4, m=2, \alpha=0.05$, and $\alpha_{1}=\alpha_{2}=\alpha_{3}=$ $\alpha_{4}=1$, and the other corresponding parameters are shown in Table 1.

Solution. When $n_{1}=0, n_{2}=4, \Omega_{21}=5.9931, \Omega_{22}=$ $7.6125, \Omega_{23}=7.2500, \Omega_{24}=5$, then the optimal schedule on machine $M_{2}$ is $\left[J_{4}, J_{1}, J_{2}, J_{3}\right]$ and $Z_{1}=155.2178$.

When $n_{1}=1, n_{2}=3, \Omega_{11}=2, \Omega_{21}=4.4600, \Omega_{22}=$ 5.2000, $\Omega_{23}=4$, the optimal schedule on machine $M_{1}$ is $\left[J_{3}\right]$ and on machine $M_{2}$ is $\left[J_{4}, J_{1}, J_{2}\right]$, and $Z_{1}=102.7967$.

When $n_{1}=2, n_{2}=2, \Omega_{11}=3.1500, \Omega_{12}=3, \Omega_{21}=$ 3.1500, $\Omega_{22}=3$, the optimal schedule on machine $M_{1}$ is $\left[J_{2}, J_{3}\right]$ and on machine $M_{2}$ is $\left[J_{4}, J_{1}\right]$, and $Z_{1}=99.9928$.

When $n_{1}=3, n_{2}=1, \Omega_{11}=4.4600, \Omega_{12}=5.2000, \Omega_{13}=$ $4, \Omega_{21}=2$, the optimal schedule on machine $M_{1}$ is $\left[J_{2}, J_{3}, J_{4}\right]$ and on machine $M_{2}$ is $\left[J_{1}\right]$, and $Z_{1}=115.8243$.

When $n_{1}=4, n_{2}=0, \Omega_{11}=5.9931, \Omega_{12}=7.6125, \Omega_{13}=$ 7.2500, $\Omega_{14}=5$, the optimal schedule on machine $M_{1}$ is $\left[J_{4}, J_{3}, J_{2}, J_{1}\right]$, and $Z_{1}=168.1833$.

Hence, the optimal schedule on machine $M_{1}$ is $\left[J_{2}, J_{3}\right]$ and on machine $M_{2}$ is $\left[J_{4}, J_{1}\right], u_{1[1]}^{*}=4, u_{1[2]}^{*}=3, u_{2[1]}^{*}=2, u_{2[2]}^{*}=$ 3 and optimal cost is $Z_{1}=99.9928$.

4. Problem $R m \mid p_{i j}=a_{i j} r^{b_{i j}}+\alpha t-$

$$
\begin{aligned}
& \theta_{i j} u_{i j} \mid \alpha_{1} \sum_{i=1}^{m} L_{i}+\alpha_{2} \sum_{i=1}^{m} T W_{i}+ \\
& \alpha_{3} \sum_{i=1}^{m} T A D W_{i}+\alpha_{4} \sum_{i=1}^{m} \sum_{j=1}^{n_{i}} G_{i j} u_{i j}
\end{aligned}
$$

Similar to the analysis of the problem $R m \mid p_{i j}=a_{i j} b^{b_{i j}}+$ $\alpha t-\theta_{i j} u_{i j} \mid \alpha_{1} \sum_{i=1}^{m} L_{i}+\alpha_{2} \sum_{i=1}^{m} T C_{i}+\alpha_{3} \sum_{i=1}^{m} T A D C_{i}+$ $\alpha_{4} \sum_{i=1}^{m} \sum_{j=1}^{n_{i}} G_{i j} u_{i j}$ presented in the previous section, if we substitute $W_{i[j]}=\sum_{l=1}^{j-1} p_{i[l]}, T W_{i}=\sum_{j=1}^{n_{i}} W_{i[j]}$, and $T A D W_{i}$ $=\sum_{j=1}^{n_{i}} j\left(n_{i}-j\right) p_{i[j]}$ (Bagchi [36]) into (7), we have

$$
\begin{aligned}
Z_{2}(\pi, u)= & \alpha_{1} \sum_{i=1}^{m} \sum_{j=1}^{n_{i}} p_{i[j]}+\alpha_{2} \sum_{i=1}^{m} \sum_{j=1}^{n_{i}}\left(n_{i}-j\right) p_{i[j]} \\
& +\alpha_{3} \sum_{i=1}^{m} \sum_{j=1}^{n_{i}} j\left(n_{i}-j\right) p_{i[j]}+\alpha_{4} \sum_{i=1}^{m} \sum_{j=1}^{n_{i}} G_{i[j]} u_{i[j]} \\
= & \sum_{i=1}^{m} \sum_{j=1}^{n_{i}} \Psi_{i j} a_{i[j]} b_{i[j]} \\
& +\sum_{i=1}^{m} \sum_{j=1}^{n_{i}}\left(\alpha_{4} G_{i[j]}-\beta_{i[j]} \Psi_{i j}\right) u_{i[j]},
\end{aligned}
$$

where $v_{i j}=\alpha_{1}+\alpha_{2}\left(n_{i}-j\right)+\alpha_{3} j\left(n_{i}-j\right)$,

$$
\Psi_{i 1}=v_{i 1}+\alpha v_{i 2}+\alpha(1+\alpha) v_{i 3}+\cdots+\alpha(1+\alpha)^{n_{i}-2} v_{i n_{i}},
$$

TABLE 1: The date of Example 4.

\begin{tabular}{ccccc}
\hline$J_{j}$ & $J_{1}$ & $J_{2}$ & $J_{3}$ & $J_{4}$ \\
\hline$a_{1 j}$ & 16 & 20 & 18 & 14 \\
$a_{2 j}$ & 17 & 19 & 20 & 12 \\
$b_{1 j}$ & -0.35 & -0.21 & -0.25 & -0.31 \\
$b_{2 j}$ & -0.23 & -0.31 & -0.27 & -0.25 \\
$\theta_{1 j}$ & 2 & 3 & 4 & 5 \\
$\theta_{2 j}$ & 4 & 5 & 2 & 3 \\
$\bar{u}_{1 j}$ & 3 & 4 & 3 & 1 \\
$\bar{u}_{2 j}$ & 3 & 2 & 5 & 2 \\
$G_{1 j}$ & 6 & 4 & 2 & 3 \\
$G_{2 j}$ & 3 & 7 & 5 & 4 \\
\hline
\end{tabular}

$$
\begin{aligned}
\Psi_{i 2} & =v_{i 2}+\alpha v_{i 3}+\alpha(1+\alpha) v_{i 4}+\cdots+\alpha(1+\alpha)^{n_{i}-3} v_{i n_{i}}, \\
\Psi_{i 3} & =v_{i 3}+\alpha v_{i 4}+\alpha(1+\alpha) v_{i 5}+\cdots+\alpha(1+\alpha)^{n_{i}-4} v_{i n_{i}}, \\
& \vdots \\
\Psi_{i, n_{i}-1} & =v_{i, n_{i}-1}+\alpha v_{i n_{i}}, \\
\Psi_{i n_{i}} & =v_{i i_{i}} .
\end{aligned}
$$

Applying a similar analysis in the previous section, we have the following results.

Lemma 5. For a given sequence, the optimal resource allocation of the problem Rm $\left|p_{i j}=a_{i j} r^{b_{i j}}+\alpha t-\theta_{i j} u_{i j}\right| \alpha_{1} \sum_{i=1}^{m} L_{i}+$ $\alpha_{2} \sum_{i=1}^{m} T W_{i}+\alpha_{3} \sum_{i=1}^{m} T A D W_{i}+\alpha_{4} \sum_{i=1}^{m} \sum_{j=1}^{n_{i}} G_{i j} u_{i j}$ can be determined by

$$
u_{i[j]}^{*}= \begin{cases}\bar{u}_{i[j]}, & \text { if } \alpha_{4} G_{i[j]}-\beta_{i[j]} \Psi_{i j}<0, \\ u_{i[j]} \in\left[0, \bar{u}_{i[j]}\right], & \text { if } \alpha_{4} G_{i[j]}-\beta_{i[j]} \Psi_{i j}=0, \\ 0, & \text { if } \alpha_{4} G_{i[j]}-\beta_{i[j]} \Psi_{i j}>0,\end{cases}
$$

where $u_{i[j]}^{*}, i=1,2, \ldots, m ; j=1,2, \ldots, n_{i}$ represents the optimal resource allocation of the job in position $j$ on machine $M_{i}$.

Lemma 6. For the problem $R m\left|p_{i j}=a_{i j} r^{b_{i j}}+\alpha t-\theta_{i j} u_{i j}\right|$ $\alpha_{1} \sum_{i=1}^{m} L_{i}+\alpha_{2} T W_{i}+\alpha_{3} T A D W_{i}+\alpha_{4} \sum_{i=1}^{m} \sum_{j=1}^{n_{i}} G_{i j} u_{i j}$, if the vector $P(n, m)=\left(n_{1}, n_{2}, \ldots, n_{m}\right)$ is given, the optimal sequence can be determined by solving the following assignment problem:

$\operatorname{Min} Z=\sum_{i=1}^{m} \sum_{r=1}^{n_{i}} \sum_{j=1}^{n} \theta_{i j r} x_{i j r}$

subject to (13), (14) and (15), 
where

$$
\begin{aligned}
& \theta_{i j r} \\
& = \begin{cases}\Psi_{i r} a_{i j} r^{b_{i j}}, & \text { if } \delta_{4} G_{i j}-\beta_{i j} \Psi_{i r} \geq 0, \\
\Omega_{i r} a_{i j} r^{b_{i j}}+\left(\alpha_{4} G_{i j}-\beta_{i j} \Psi_{i r}\right) m_{i j}, & \text { if } \alpha_{4} G_{i j}-\beta_{i j} \Psi_{i r}<0 .\end{cases}
\end{aligned}
$$

Theorem 7. The problem $R m\left|p_{i j}=a_{i j} b^{b_{i j}}+\alpha t-\theta_{i j} u_{i j}\right|$ $\alpha_{1} \sum_{i=1}^{m} L_{i}+\alpha_{2} T W_{i}+\alpha_{3} T A D W_{i}+\alpha_{4} \sum_{i=1}^{m} \sum_{j=1}^{n_{i}} G_{i j} u_{i j}$ can be solved in $\mathrm{O}\left(n^{m+2}\right)$ time; that is, the problem is polynomially solvable because $m$ is a constant.

The optimal solution for the problem $R m \mid p_{i j}=a_{i j} r^{b_{i j}}+$ $\alpha t-\theta_{i j} u_{i j} \mid \alpha_{1} \sum_{i=1}^{m} L_{i}+\alpha_{2} T W_{i}+\alpha_{3} T A D W_{i}+\alpha_{4} \sum_{i=1}^{m} \sum_{j=1}^{n_{i}} G_{i j} u_{i j}$ can be obtained by the following algorithm.

\section{Algorithm 8. Consider the following.}

Step 1. For all the possible vectors $\left(n_{1}, n_{2}, \ldots, n_{m}\right)$, solve the assignment problems ((20), (13)-(15), (21)). Then, obtain the optimal schedule and the corresponding total cost $\alpha_{1} \sum_{i=1}^{m} L_{i}+\alpha_{2} \sum_{i=1}^{m} T W_{i}+\alpha_{3} \sum_{i=1}^{m} T A D W_{i}+\alpha_{4} \sum_{i=1}^{m} \sum_{j=1}^{n_{i}} G_{i j} u_{i j}$ for each possible vector $\left(n_{1}, n_{2}, \ldots, n_{m}\right)$.

Step 2. The optimal solution for the problem is the one with the minimum value of the total $\operatorname{cost} \alpha_{1} \sum_{i=1}^{m} L_{i}+\alpha_{2} \sum_{i=1}^{m} T W_{i}+$ $\alpha_{3} \sum_{i=1}^{m} T A D W_{i}+\alpha_{4} \sum_{i=1}^{m} \sum_{j=1}^{n_{i}} G_{i j} u_{i j}$.

Step 3. Calculate the optimal resources allocation by using (19).

The following example illustrates the working of Algorithm 8 for the problem $R m\left|p_{i j}=a_{i j} r^{b_{i j}}+\alpha t-\theta_{i j} u_{i j}\right|$ $\alpha_{1} \sum_{i=1}^{m} L_{i}+\alpha_{2} \sum_{i=1}^{m} T W_{i}+\alpha_{3} \sum_{i=1}^{m} T A D W_{i}+\alpha_{4} \sum_{i=1}^{m} \sum_{j=1}^{n_{i}} G_{i j} u_{i j}$.

Example 9. The same date in Example 4 is used.

Solution. When $n_{1}=0, n_{2}=4, \Psi_{21}=7.6676, \Psi_{22}=7.3025$, $\Psi_{23}=5.0500, \Psi_{24}=1$, then the optimal schedule on machine $M_{2}$ is $\left[J_{4}, J_{1}, J_{2}, J_{3}\right]$ and $Z_{2}=126.7347$.

When $n_{1}=1, n_{2}=3, \Psi_{11}=1, \Psi_{21}=5.2525, \Psi_{22}=$ $4.0500, \Psi_{23}=1$, the optimal schedule on machine $M_{1}$ is $\left[J_{3}\right]$ and on machine $M_{2}$ is $\left[J_{4}, J_{1}, J_{2}\right]$, and $Z_{2}=84.13484$.

When $n_{1}=2, n_{2}=2, \Psi_{11}=3.0500, \Psi_{12}=1, \Psi_{21}=$ $3.0500, \Psi_{22}=1$, the optimal schedule on machine $M_{1}$ is $\left[J_{3}, J_{4}\right]$ and on machine $M_{2}$ is $\left[J_{1}, J_{2}\right]$, and $Z_{2}=73.1692$.

When $n_{1}=3, n_{2}=1, \Psi_{11}=5.2525, \Psi_{12}=4.0500, \Psi_{13}=$ $1, \Psi_{21}=1$, the optimal schedule on machine $M_{1}$ is $\left[J_{3}, J_{4}, J_{2}\right]$ and on machine $M_{2}$ is $\left[J_{1}\right]$, and $Z_{2}=95.8810$.

When $n_{1}=4, n_{2}=0, \Psi_{11}=7.6676, \Psi_{12}=7.3025, \Psi_{13}=$ $5.0500, \Psi_{14}=1$, the optimal schedule on machine $M_{1}$ is $\left[J_{4}, J_{3}, J_{2}, J_{1}\right]$, and $Z_{2}=144.1351$.

Hence, the optimal schedule on machine $M_{1}$ is $\left[J_{3}, J_{4}\right]$ and on machine $M_{2}$ is $\left[J_{1}, J_{2}\right], u_{1[1]}^{*}=3, u_{1[2]}^{*}=1, u_{2[1]}^{*}=3, u_{2[2]}^{*}=$ 0 and optimal cost is $Z_{2}=73.1692$.

\section{Conclusions}

In this paper, we have studied the problem of scheduling $n$ jobs on $m$ unrelated parallel machines with simultaneous consideration of learning effect, deteriorating jobs, and controllable processing times. We provide an $O\left(n^{m+2}\right)$ time algorithm for the problems $R m\left|p_{i j}=a_{i j} r^{b_{i j}}+\alpha t-\theta_{i j} u_{i j}\right|$ $\alpha_{1} \sum_{i=1}^{m} L_{i}+\alpha_{2} \sum_{i=1}^{m} T C_{i}+\alpha_{3} \sum_{i=1}^{m} T_{A D C}+\alpha_{4} \sum_{i=1}^{m} \sum_{j=1}^{n_{i}} G_{i j} u_{i j}$ and $R m\left|p_{i j}=a_{i j} r^{b_{i j}}+\alpha t-\theta_{i j} u_{i j}\right| \alpha_{1} \sum_{i=1}^{m} L_{i}+\alpha_{2} \sum_{i=1}^{m} T W_{i}+$ $\alpha_{3} \sum_{i=1}^{m} T A D W_{i}+\alpha_{4} \sum_{i=1}^{m} \sum_{j=1}^{n_{i}} G_{i j} u_{i j}$, respectively. The algorithms can also be easily applied to the cases $b_{i j}>0$ (aging effect) and $\alpha<0$. Future research may focus on similar problems with more general processing time model and extend the problems to flow shop machine settings.

\section{Conflict of Interests}

The authors declare that there is no conflict of interests regarding the publication of this paper.

\section{Acknowledgments}

The authors are grateful for three anonymous referees for their helpful comments on earlier version of the paper. This research was supported by the National Natural Science Foundation of China (Grant nos. 11001181 and 71271039), the New Century Excellent Talents in University (NCET13-0082), the Changjiang Scholars and Innovative Research Team in University (IRT1214), and the Fundamental Research Funds for the Central Universities (DUT14YQ211).

\section{References}

[1] B. Alidaee and N. K. Womer, "Scheduling with time dependent processing times: review and extensions," Journal of the Operational Research Society, vol. 50, no. 7, pp. 711-720, 1999.

[2] T. C. E. Cheng, Q. Ding, and B. M. T. Lin, "A concise survey of scheduling with time-dependent processing times," European Journal of Operational Research, vol. 152, no. 1, pp. 1-13, 2004.

[3] S. Gawiejnowicz, Time-Dependent Scheduling, Springer, Berlin, Germany, 2008.

[4] D. Biskup, "A state-of-the-art review on scheduling with learning effects," European Journal of Operational Research, vol. 188, no. 2, pp. 315-329, 2008.

[5] D. Shabtay and G. Steiner, "A survey of scheduling with controllable processing times," Discrete Applied Mathematics, vol. 155, no. 13, pp. 1643-1666, 2007.

[6] J. Bai, Z.-R. Li, and X. Huang, "Single-machine group scheduling with general deterioration and learning effects," Applied Mathematical Modelling, vol. 36, no. 3, pp. 1267-1274, 2012.

[7] J. Bai, M.-Z. Wang, and J.-B. Wang, "Single machine scheduling with a general exponential learning effect," Applied Mathematical Modelling, vol. 36, no. 2, pp. 829-835, 2012.

[8] T. C. E. Cheng, C.-J. Hsu, and D.-L. Yang, "Unrelated parallelmachine scheduling with deteriorating maintenance activities," Computers and Industrial Engineering, vol. 60, no. 4, pp. 602605, 2011. 
[9] M. Gorczyca and A. Janiak, "Resource level minimization in the discrete-continuous scheduling," European Journal of Operational Research, vol. 203, no. 1, pp. 32-41, 2010.

[10] C.-J. Hsu and D.-L. Yang, "Unrelated parallel-machine scheduling with position-dependent deteriorating jobs and resourcedependent processing time," Optimization Letters, vol. 8, pp. 519-531, 2014.

[11] X. Huang and M.-Z. Wang, "Parallel identical machines scheduling with deteriorating jobs and total absolute differences penalties," Applied Mathematical Modelling, vol. 35, no. 3, pp. 1349-1353, 2011.

[12] W.-C. Lee, C.-C. Wu, Y.-H. Chung, and H.-C. Liu, "Minimizing the total completion time in permutation flow shop with machine-dependent job deterioration rates," Computers and Operations Research, vol. 36, no. 6, pp. 2111-2121, 2009.

[13] Y. Leyvand, D. Shabtay, and G. Steiner, "A unified approach for scheduling with convex resource consumption functions using positional penalties," European Journal of Operational Research, vol. 206, no. 2, pp. 301-312, 2010.

[14] H. Nian and Z. Mao, "Single-machine scheduling with job rejection, deteriorating effects, and deteriorating maintenance activities," Mathematical Problems in Engineering, vol. 2013, Article ID 389120, 9 pages, 2013.

[15] D. Shabtay and G. Steiner, "The single-machine earlinesstardiness scheduling problem with due date assignment and resource-dependent processing times," Annals of Operations Research, vol. 159, no. 1, pp. 25-40, 2008.

[16] J.-B. Wang, L. Liu, and C. Wang, "Single machine SLK/DIF due window assignment problem with learning effect and deteriorating jobs," Applied Mathematical Modelling, vol. 37, pp. 8394-8400, 2013.

[17] J.-B. Wang and M.-Z. Wang, "Worst-case analysis for flow shop scheduling problems with an exponential learning effect," Journal of the Operational Research Society, vol. 63, no. 1, pp. 130-137, 2012.

[18] J.-B. Wang and J.-J. Wang, "Flowshop scheduling with a general exponential learning effect," Computers \& Operations Research, vol. 43, pp. 292-308, 2014.

[19] J.-B. Wang, M.-Z. Wang, and P. Ji, "Scheduling jobs with processing times dependent on position, starting time and allotted resource," Asia-Pacific Journal of Operational Research, vol. 29, Article ID 1250030, 2012.

[20] J.-B. Wang, X.-Y. Wang, L.-H. Sun, and L.-Y. Sun, "Scheduling jobs with truncated exponential learning functions," Optimization Letters, vol. 7, no. 8, pp. 1857-1873, 2011.

[21] D. Wang, M.-Z. Wang, and J.-B. Wang, "Single-machine scheduling with learning effect and resource-dependent processing times," Computers and Industrial Engineering, vol. 59, no. 3, pp. 458-462, 2010.

[22] C.-M. Wei, J.-B. Wang, and P. Ji, "Single-machine scheduling with time-and-resource-dependent processing times," Applied Mathematical Modelling, vol. 36, no. 2, pp. 792-798, 2012.

[23] C.-C. Wu and W.-C. Lee, "Single-machine and flowshop scheduling with a general learning effect model," Computers and Industrial Engineering, vol. 56, no. 4, pp. 1553-1558, 2009.

[24] C.-C. Wu and C.-L. Liu, "Minimizing the makespan on a single machine with learning and unequal release times," Computers and Industrial Engineering, vol. 59, no. 3, pp. 419-424, 2010.
[25] D.-L. Yang and W.-H. Kuo, "Some scheduling problems with deteriorating jobs and learning effects," Computers and Industrial Engineering, vol. 58, no. 1, pp. 25-28, 2010.

[26] S.-J. Yang and D.-L. Yang, "Single-machine group scheduling problems under the effects of deterioration and learning," Computers and Industrial Engineering, vol. 58, no. 4, pp. 754758, 2010.

[27] D. L. Yang, T. C. E. Cheng, and S. J. Yang, "Parallelmachine scheduling with controllable processing times and rate-modifying activities to minimise total cost involving total completion time and job compressions," International Journal of Production Research, vol. 52, no. 4, pp. 1133-1141, 2014.

[28] Y. Yin, T. C. E. Cheng, C. C. Wu, and S. R. Cheng, "Singlemachine common due-date scheduling with batch delivery costs and resource-dependent processing times," International Journal of Production Research, vol. 51, pp. 5083-5099, 2013.

[29] Y. Yin, T. C. E. Cheng, C. C. Wu, and S. R. Cheng, "Singlemachine due window assignment and scheduling with a common flow allowance and controllable job processing time," Journal of the Operational Research Society, vol. 65, pp. 1-13, 2014.

[30] N. Yin, L. Kang, T. C. Sun, C. Yue, and X. R. Wang, "Unrelated parallel machines scheduling with deteriorating jobs and resource dependent processing times," Applied Mathematical Modelling, 2014.

[31] N. Yin, L. Kang, and X. Y. Wang, "Single-machine group scheduling with processing times dependent on position, starting time and allotted resource," Applied Mathematical Modelling, 2014.

[32] C. L. Zhao and H. Y. Tang, "Single machine scheduling problems with general positiondependent processing times and past-sequence-dependent delivery times," Journal of Applied Mathematics and Computing, vol. 45, no. 1-2, pp. 259-274, 2014.

[33] G. Mosheiov and J. B. Sidney, "Scheduling with general jobdependent learning curves," European Journal of Operational Research, vol. 147, no. 3, pp. 665-670, 2003.

[34] R. L. Graham, E. L. Lawler, J. K. Lenstra, and A. H. G. R. Kan, "Optimization and approximation in deterministic sequencing and scheduling: a survey," Annals of Discrete Mathematics, vol. 5, pp. 287-326, 1979.

[35] J. J. Kanet, "Minimizing variation of flow time in single machine systems," Management Science, vol. 27, no. 12, pp. 1453-1464, 1981.

[36] U. Bagchi, "Simultaneous minimization of mean and variation of flow time and waiting time in single machine systems," Operations Research, vol. 37, no. 1, pp. 118-125, 1989. 


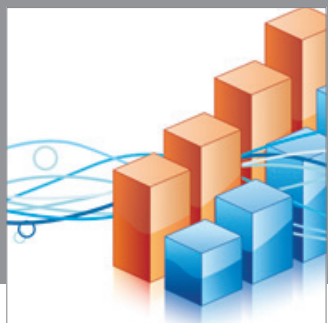

Advances in

Operations Research

mansans

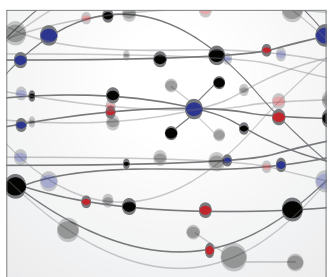

The Scientific World Journal
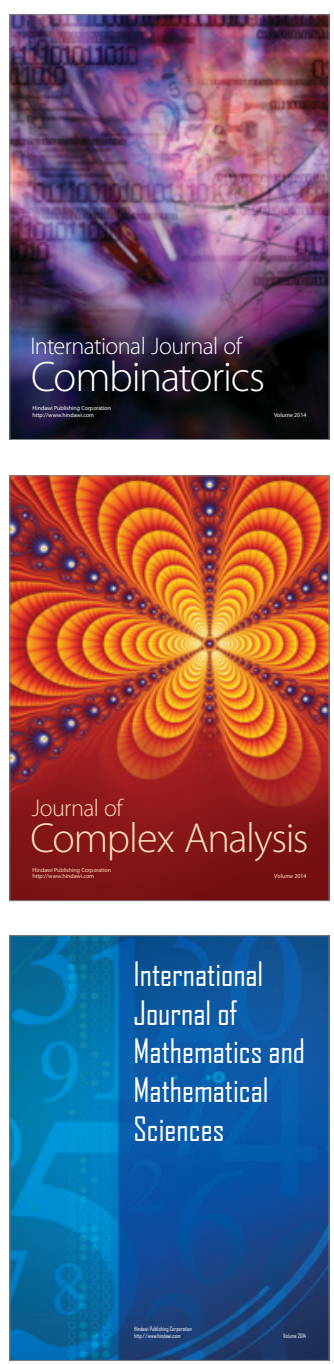
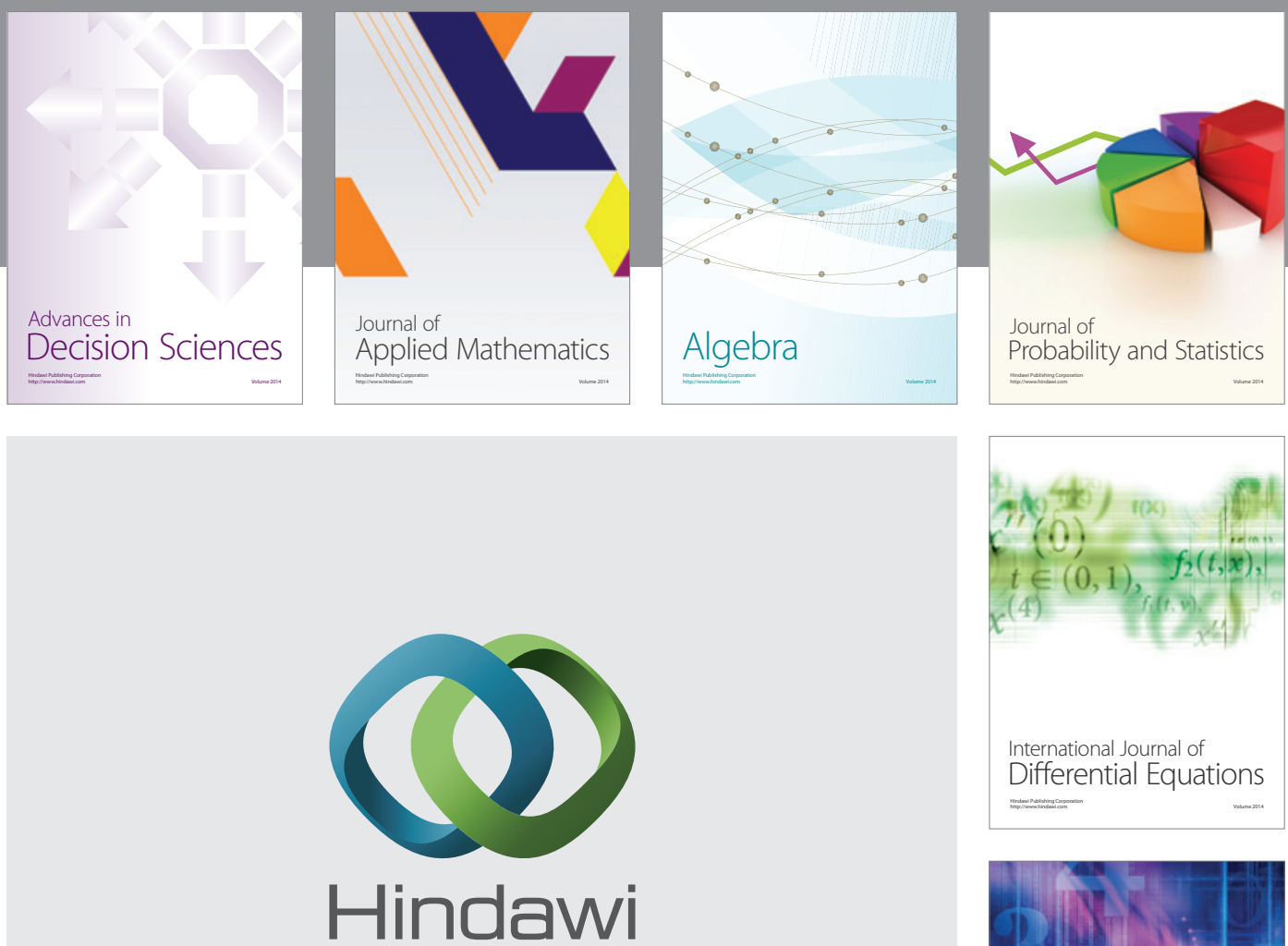

Submit your manuscripts at http://www.hindawi.com
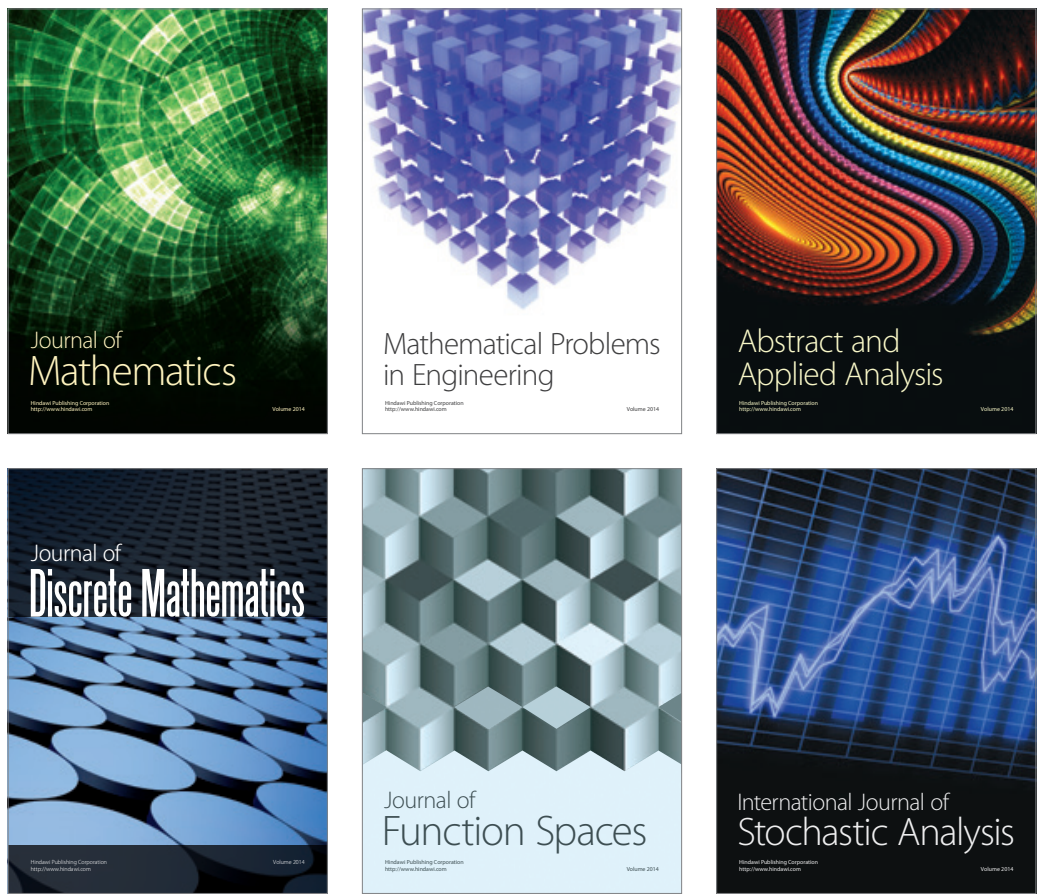

Journal of

Function Spaces

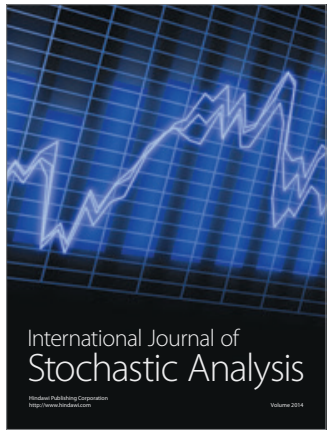

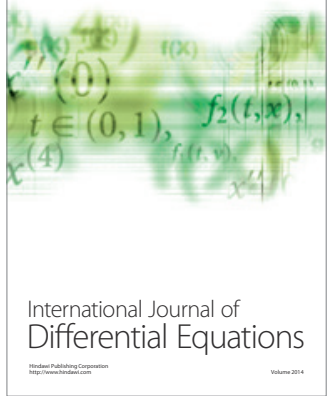
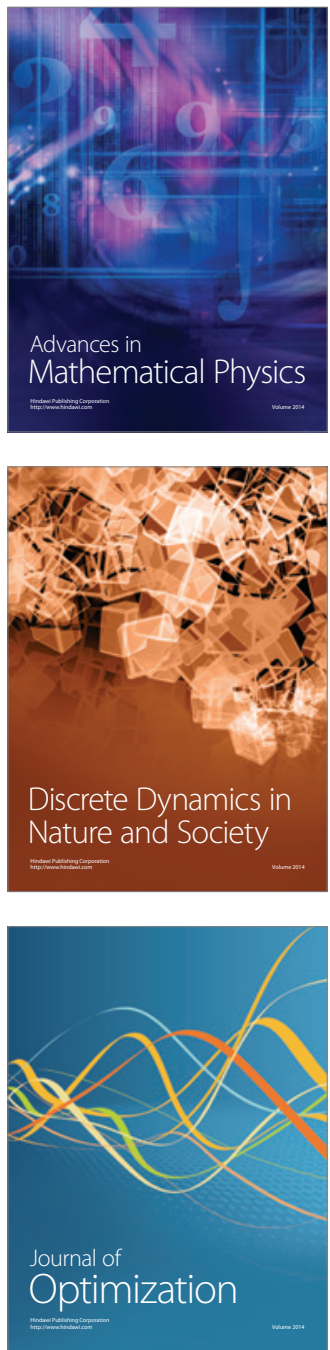